\title{
Farmácia hospitalar como oportunidade para a gestão de design no trabalho de prevenção do erro de medicação: estudo em uma realidade brasileira
}

\section{Hospital pharmacy department as an opportunity to design management for medicine error prevention: a study of a brazilian reality}

\author{
Blum, Arina ${ }^{a}$; Merino - Schmidt, Giselle ${ }^{b}$ \& Merino - Díaz, Eugenio Andrés \\ a Programa de Pós-graduação em Design, Universidade Federal de Santa Catarina, Brasil - arinablum@gmail.com \\ ${ }^{\text {b }}$ Programa de Pós-graduação em Design, Universidade Federal de Santa Catarina, Brasil \& Programa de Pós- \\ graduação em Design, Universidade Estadual de Santa Catarina, Brasil - gisellemerino@gmail.com \\ ${ }^{c}$ Programa de Pós-graduação em Design, Universidade Federal de Santa Catarina, Brasil \& Programa de Pós- \\ graduação em Engenharia de Produção, Brasil - eugenio.merino@ufsc.br
}

\begin{abstract}
Resumo
O erro de medicação é um problema do qual nenhum hospital está imune. O trabalho para a prevenção do erro deve, portanto, ser uma constante que visa, em última análise, a segurança do paciente. Um antecedente importante para reduzir a probabilidade da ocorrência do erro é criação de uma cultura de segurança em todo o sistema de medicação. (Werner, Nelson e Boehm-Davis, 2012; Anvisa, 2010; Otero López et al., 2008b; Schneider, 2007; entre outros). A gestão de design pode agir, assim, contribuindo com a resolução de problemas de forma ordenada e lógica (Borja de Mozota, Klöpsch e Xavier da Costa, 2011) e cooperando para a eficiência estratégica das inter-relações (Best, 2012). Neste sentido, deu-se a questão de pesquisa: em que setor do hospital pode-se iniciar um trabalho de gestão de design que venha a contribuir para a prevenção do erro de medicação? O objetivo traçado pela pesquisa foi de identificar um setor do hospital onde ações de design possam ser implantadas para, de forma estratégica, prevenir o erro de medicação. Para tanto, foi realizada uma pesquisa sistemática, com foco em um levantamento bibliográfico exploratório, de natureza básica. Primeiramente foram abarcados dados qualitativos sobre as características do erros de medicação e a sua classificação segundo a literatura. Posteriormente houve o mapeamento dos setores envolvidos no sistema de medicação e o apontamento de ações onde a gestão de design pode atuar como apoio à prevenção do erro. O resultado indicou a farmácia hospitalar como um setor estratégico e de oportunidade para a atuação da gestão de design. Ficou evidenciado que, ali, o trabalho de prevenção tem sido efetivado essencialmente por profissionais da área da saúde que tem o domínio sobre as formas nas quais o erro de medicação pode ser evitado, porém desconhecem o campo do design em sua abrangência e, portanto, não utilizam as possibilidades dessa área. Outros resultados concentram-se no apontamento, ainda inicial, de possibilidades nos niveis operacional, tático e estratégico do design que cooperam para a prevenção do erro de medicação. Entre elas, citam-se melhorias nas identificações de produtos fracionados e armazenados, organização do espaço e otimização do trabalho. Esta pesquisa foi parte embasadora de um projeto que
\end{abstract}


Farmácia hospitalar como oportunidade para a gestão de design no trabalho de prevenção do erro de medicação: estudio em uma realidade brasileira.

está em prática em um laboratório de design de uma universidade brasileira. Ocorre a partir do trabalho de uma equipe multidisciplinar, que conta com pesquisadores designers, em parceria com profissionais da área da saúde atuantes em um hospital público psiquiátrico.

Palavras-chave: design, gestão, sistema, hospital, medicação.

\begin{abstract}
Medication error is a potential risk in any hospital. Preventing medication error must therefore be a continuous work, aiming the safety of the patients. The creation of a safety culture throughout all the hospital medication system is an important step to reduce probability of error. (Werner, Nelson e Boehm-Davis, 2012; Anvisa, 2010; Otero López et al., 2008b; Schneider, 2007; among others). Thus, design management may act in order to contribute solving problems in an ordered and logic way (Borja de Mozota, Klöpsch and Xavier da Costa, 2011) and to collaborate with the strategic efficiency of interrelations (Best, 2012). This research aims to identify a department in the hospital where design actions might take place to prevent medication errors in a strategic way. Therefore, a systematic research was conducted, focusing on basic exploratory bibliographic survey. At first, qualitative data about the characteristics of medication errors were gathered and classified, based on literature. Then the departments evolved on the medication system were charted to point where design management might act to support error prevention. The results indicated the hospital pharmacy as an opportune and strategic department for the actions of design management. It was evidenced that at the hospital pharmacy, prevention work has been essentially performed by health professionals who are proficient at the ways to avoid errors, but disregard the field of design in all its range, and therefore, they do not use the possibilities of it. Other results focus on pointing, still early, possibilities on operational, tactical, and strategic levels of design that cooperate to medication error prevention. Among them, better identification on fractionated and stocked products, space organization, and optimization of work processes were reported. This research was the basis of a project that is being conducted on a design laboratory in a Brazilian university. It occurs from a multidisciplinary team, counting with design researchers in partnership with health professionals of a public psychiatric hospital.
\end{abstract}

Keywords: design, management, system, hospital, medication. 


\section{Introdução}

Os erros de medicação, segundo Mendes et al. (2014), são importantes causas de morbidade e mortalidade. Os autores apontam que tais erros causam a morte de 1 (um) a cada 131 (cento e trinta e um) pacientes ambulatoriais e 1 (um) a cada 854 (oitocentos e cinquenta e quatro) pacientes internados, o que consiste numa taxa de erros de medicação que varia entre 4,8\% e 5,3\%. Pesquisadores que estudaram o tema em hospitais públicos brasileiros, chegaram a identificar problemas na administração de $30 \%$ dos casos (Moreira Reis et al., 2010).

Os erros estão ligados a diversos fatores relacionados ao paciente, aos profissionais de saúde e ao medicamento, como a semelhança de embalagem e dos nomes dos produtos e a maneira de identificá-los nos processos intrínsecos ao sistema de medicação. Podem ser ocasionados por problemas de prescrição, por omissão, por dispensação equivocada, por administração de dose errada ou dose farmacêutica imprópria, por preparação inadequada, entre outros. (MENDES et al., 2014; Almeida Lopes et al., 2012; Moreira Reis et al., 2010; Azevedo Anacleto et al., 2010)

Nesse contexto e, ainda, considerando relatos trazidos pela mídia brasileira onde chamaram a atenção a presença de erros como armazenamento de medicamentos em local equivocado, relatos de sobrecarga de trabalho vivida pelo professional da saúde e trocas decorrentes de extrema semelhança de embalagens e rótulos de medicamentos (TEIXEIRA, 2011; G1, 2010; CAVALLARI, 2010), apresenta-se a pesquisa concentrada na seguinte questão: em que setor do hospital pode-se iniciar um trabalho de gestão de design que venha a contribuir para a prevenção do erro de medicação?

A pesquisa partiu do princípio de que o erro de medicação é um aspecto que nenhum hospital está imune (Werner, Nelson e Boehm-Davis, 2012, entre outros) e que existem protocolos indicando caminhos para a prevenção desse tipo de erro (Anvisa, 2010; Otero López et al., 2008b). Também considerou que a gestão de design, enquanto processo sistemático, ou seja, de resolução de problemas de forma ordenada e lógica (MOZOTA; KLÖPSCH; COSTA, 2011), pode contribuir para a questão da prevenção do erro de medicação. Isso porque o contexto do sistema de medicação hospitalar tem características que demandam um trabalho orientado a uma abordagem global que, ao mesmo tempo, considera partes individualizadas. A gestão de design, justamente, apresenta-se sob a perspectiva de uma visão do todo unificado, conectado a partir da eficiência das inter-relações e interdependências das partes individuais que o compõem (Best, 2012).

Neste sentido, o artigo apresenta a pesquisa que delineou-se no objetivo geral de identificar um setor do hospital onde ações de design possam ser implantadas para, de forma estratégica, prevenir o erro de medicação. Como objetivos específicos, visou-se realizar um levantamento sistemático para verificar os indicativos da literatura neste contexto e compreender como se dá o sistema de medicação na realidade brasileira.

Para tanto, foi realizada uma pesquisa sistemática, com foco em um levantamento bibliográfico exploratório, de natureza básica. Primeiramente foram abarcados dados qualitativos sobre as características do erros de medicação e a sua classificação segundo a literatura. Posteriormente houve o mapeamento dos setores envolvidos no sistema de medicação, a visita para conhecimento da realidade de um hospital e o apontamento de ações onde a gestão de design pode atuar como apoio à prevenção do erro. 
Farmácia hospitalar como oportunidade para a gestão de design no trabalho de prevenção do erro de medicação: estudio em uma realidade brasileira.

\section{Procedimentos metodológicos}

O levantamento bibliográfico exploratório ocorreu em duas etapas. A primeira, deu-se por meio da pesquisa sistemática que abrangeu, especialmente, artigos de periódicos científicos disponibilizados nas bases Ebsco Host (Ebsco-Publishing, 2015), Scielo (Fapesp et al., 2015), Science Direct (Elsevier, 2015a), Scopus (Elsevier, 2015b) e Web of Science (Thomson Reuters, 2015). Também houveram levantamentos nas bases BDTD (Ibict, 2015) e ProQuest (2015) a fim de identificar possíveis teses nesta área de interesse. A partir desta pesquisa sistemática, que utilizou procedimentos baseados no Processo de Mineração de Dados (Blum, Merino - Díaz e Merino - Schmidt, 2015), também se chegou a outras fontes sobre o tema - como registros em literatura, artigos de evento e apontamentos de estudos e de diretrizes. Algumas delas eram parte das referências trazidas pelos artigos verificados em tal revisão.

A segunda etapa do levantamento bibliográfico ocorreu após a primeira fase evidenciar um setor do hospital - a farmácia. Para a constatação de que este, de fato, se enquadrava como ponto estratégico para a gestão de design, a revisão sistemática foi ampliada. Um novo levantamento incluiu especificidades deste setor, por meio do uso de descritores e operadores booleanos (Figura 1).

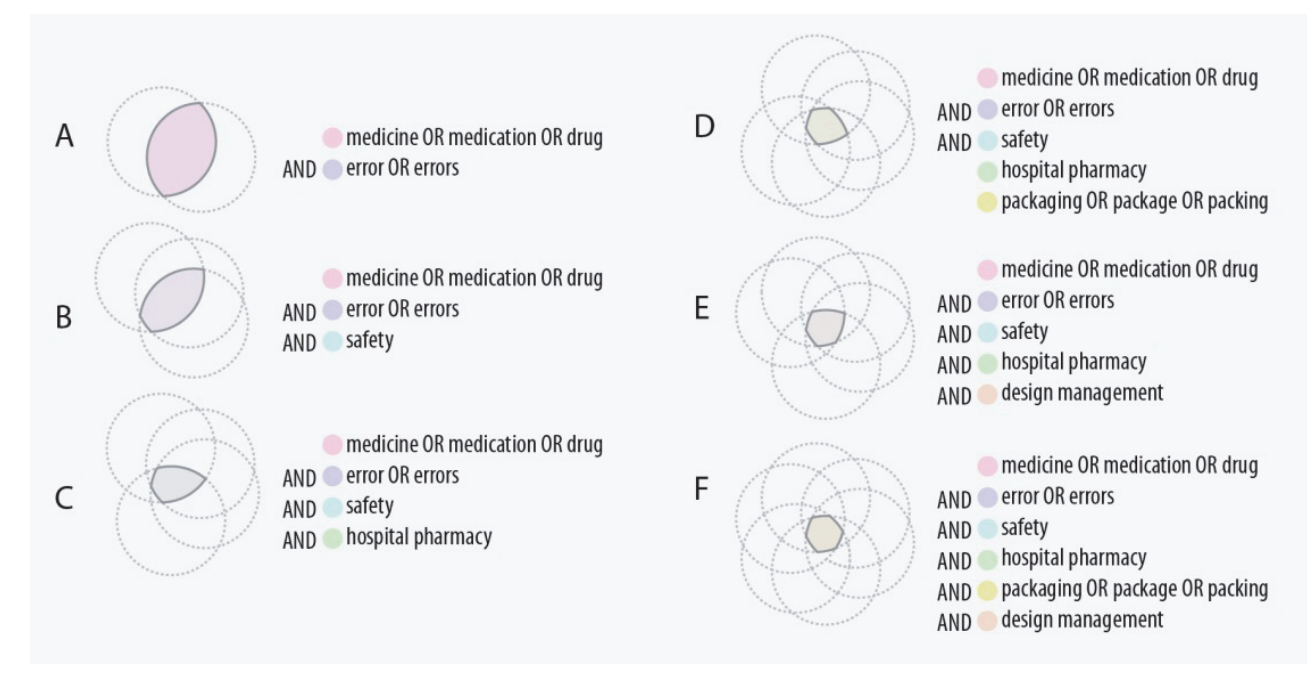

Fig.1 - Tópicos da revisão sistemática realizada na segunda etapa do levantamento bibliográfico. Fonte: os autores.

O processo baseado na Mineração de Dados é adaptado dos passos apresentados por Oliveira Rezende et al. (2005): (i) Conhecimento do domínio; (ii) Pré-processamento; (iii) Extração de padrões; (iv) Pósprocessamento; (v) Utilização do conhecimento. Na etapa (i) Conhecimento do domínio foram selecionadas as bases de dados que apresentavam um conjunto de informações relevantes, em termos de quantidade, frente ao interesse da pesquisa. Em (ii) Pré-processamento, os termos descritores foram estabelecidos, bem como selecionados os limitadores de pesquisa disponibilizados em cada base de dados.

A (iii) Extração de padrões ocorreu utilizando combinações expressas por meio de operadores booleanos.

O (iv) Pós-processamento envolveu a leitura e avaliação dos dados levantados e a seleção final que considerou, especialmente, a afinidade do dado no contexto da pesquisa - particularmente relevando o título, as palavras-chave e o resumo da publicação. Para (v) Utilização do conhecimento, procedeu-se à leitura dos textos selecionados e seu uso, tanto na construção da fundamentação teórica quanto como meio de se chegar a outros dados não contemplados nesta mineração. 


\section{O erro de medicação no contexto hospitalar}

O National Coordinating Council for Medication Error Reporting and Prevention (Ncc Merp, 2015, tradução nossa) define que um erro de medicação é "qualquer evento evitável que pode causar ou levar ao uso inapropriado de medicamentos ou a danos ao paciente, enquanto o medicamento está sob controle do profissional de saúde, paciente ou consumidor". Esclarece ainda que tais eventos "podem estar relacionados a práticas profissionais, produtos de saúde, procedimentos e sistemas; incluindo prescrições e ordens, rótulo, embalagem, nomenclatura, composição, dispensação, distribuição, administração, educação, monitoramento e uso".

De forma similar, o órgão regulador ligado ao Ministério da Saúde brasileiro, a Agência Nacional de Vigilância Sanitária - Anvisa (2010), coloca que erro de medicação é "qualquer evento evitável que, de fato ou potencialmente, pode levar ao uso inadequado de medicamento". Explica que "o uso inadequado pode ou não lesar o paciente, e não importa se o medicamento se encontra sob o controle de profissionais de saúde, do paciente ou do consumidor". Ainda, indica que o erro pode estar relacionado "à prática profissional, produtos usados na área da Saúde, procedimentos, problemas de comunicação", incluindose, portanto, questões como "prescrição, rótulos, embalagens, nomes, preparação, dispensação, distribuição, administração, educação, monitoramento e uso de medicamentos".

A abordagem do erro pode se dar de forma pessoal ou sistêmica (Reason, 1990). Azevedo Anacleto et al. (2010) comentam que, em geral, o sistema de saúde brasileiro adota a abordagem pessoal, quando o erro é considerado um fato ocasionado por um indivíduo que deve, portanto, ser punido com medidas disciplinares, tais como reprimendas orais ou escritas. Por outro lado, é ideal considerar a abordagem sistêmica, pois esta compreende o todo onde o erro está inserido. Os autores esclarecem que o erro é uma consequência e não uma causa, que o ser humano e os sistemas são falíveis e que mudar a condição humana está aquém de mudar o sistema e torná-lo mais seguro.

Neste sentido, a compreensão do sistema hospitalar é inerente ao entendimento do erro de medicação. No ambiente do hospital é preciso considerar que existem dinâmicas próprias afetadas por: imprevistos e emergências que exigem complexas tomadas de decisão; atividades desenvolvidas em coletivo e divididas em turnos de trabalho e numa série de locais ou setores; intervenções diretamente sobre o ser humano, com implicações psíquicas e sociais. O erro, desta forma, é parte integrante de um conjunto de fatores que caracterizam o hospital como "ao mesmo tempo o lugar de um futuro cada vez mais eficiente e eficaz e o local de atendimento do sofrimento humano". (Martin, Gadbois, 2007, p. 512)

Embora nem todos os erros possam ser classificados como sistêmicos - já que há comportamentos individuais que levam ao estado de risco (Azevedo Anacleto et al., 2010), a abordagem no sentido mais global favorece que falhas no processo sejam identificadas e que, desta forma, melhorias sejam implantadas para diminuir a ocorrência desses eventos (Borges Rosa e Perini, 2003). A abordagem recomendada pela Anvisa (2010) expõe a necessidade de "uma revisão de todas as etapas do ciclo do medicamento para a identificação da cadeia de falhas, quando da investigação de um erro", já que "os erros de medicação são eventos complexos, envolvendo múltiplas etapas, procedimentos e pessoas".

O erro é passível de ocorrência em qualquer ponto do sistema de medicação. Entende-se por sistema de medicação a série de procedimentos que ocorre desde a prescrição do medicamento pelo médico até o momento da administração da medicação no paciente (Monteschi Souta, 2015; Grou Volpe, 2014; Mcleod et al., 2014; Otero López et al., 2008b e 2008b; Perufo Opitz, 2006). Neste processo, os erros mais comuns, conforme explica Maiques Juliani (2014, p. 111-112), podem ser classificados em três tipos: de prescrição, de dispensação e de administração. O erro de prescrição é "um erro de decisão ou de redação, não intencional, que pode reduzir a probabilidade de o tratamento ser efetivo ou aumentar o risco 
Farmácia hospitalar como oportunidade para a gestão de design no trabalho de prevenção do erro de medicação: estudio em uma realidade brasileira.

de lesão ao paciente". O erro de dispensação considera aqueles relacionados aos equívocos de conteúdo, de rotulagem ou de documentação. O erro de administração é aquele ocorrido no preparo e na administração do medicamento.

Para compreender as circunstâncias do erro de medicação em um sistema que perpassa por diversos processos, ações de detecção do erro podem ser estabelecidas. Otero López et al. (2008a) apontam uma classificação estruturada, que visou padronizar a detecção, análise e registro dos erros de medicação na Espanha. Este modo de classificação do erro apoiou-se na taxonomia desenvolvida pelo Ncc Merp (1998). Consiste de uma série de tópicos que envolvem, desde a caracterização do paciente e das informações gerais sobre o contexto do erro e as consequências, até pontos que precisam ser considerados para detalhá-lo, tais como características específicas do processo em que houve a ocorrência e os fatores contribuintes para tanto (Quadro 1).

\section{Quadro 1. Tipos, causas e fatores associados ao erro de medicação}

\begin{tabular}{|c|c|c|}
\hline $\begin{array}{l}\text { Tipos de erro de } \\
\text { medicação }\end{array}$ & $\begin{array}{l}\text { Causas do erro de } \\
\text { medicação }\end{array}$ & $\begin{array}{l}\text { Fatores associados ao trabalho } \\
\text { que contribuem para o erro }\end{array}$ \\
\hline $\begin{array}{l}\text { (1) Medicamento errado } \\
\text { (2) Omissão de dose ou do } \\
\text { medicamento } \\
\text { (3) Dose errada } \\
\text { (4) Frequência de administração } \\
\text { errada } \\
\text { (5) Forma farmacêutica errada } \\
\text { (6) Erro de armazenamento } \\
\text { (7) Erro de preparo, manipulação } \\
\text { e/ou acondicionamento } \\
\text { (8) Técnica de administração } \\
\text { errada } \\
\text { (9) Via de administração errada } \\
\text { (10) Velocidade de } \\
\text { administração errada } \\
\text { (11) Horário errado de } \\
\text { administraçăo } \\
\text { (12) Paciente errado } \\
\text { (13) Duraçäo do tratamento } \\
\text { errada } \\
\text { (14) Monitorização insuficiente } \\
\text { do tratamento } \\
\text { (15) Medicamento deteriorado } \\
\text { (16) Falta de adesão do paciente } \\
\text { (17) Outros tipos }\end{array}$ & $\begin{array}{l}\text { (1) Problemas de } \\
\text { comunicação / interpretação } \\
\text { (2) Confusão entre } \\
\text { nome/sobrenome de } \\
\text { pacientes } \\
\text { (3) Confusão entre nomes de } \\
\text { medicamentos (nomes } \\
\text { comerciais e princípios } \\
\text { ativos) } \\
\text { (4) Problemas na rotulagem } \\
\text { / embalagem ou } \\
\text { informaçōes do produto } \\
\text { (5) Problemas nos } \\
\text { equipamentos e dispositivos } \\
\text { de dispensação / preparação } \\
\text { / administração } \\
\text { (6) Fatores individuais } \\
\text { (7) Outras causas }\end{array}$ & $\begin{array}{l}\text { (1) Ausência ou insuficiente cumprimento de práticas } \\
\text { prioritárias de segurança } \\
\text { (2) Ausência de padronização de procedimentos ou práticas } \\
\text { assistenciais } \\
\text { (3) Ausência de protocolos ou guias clínicos atualizados de } \\
\text { tratamento ou uso de medicamentos } \\
\text { (4) Ausência ou obsolescência de fontes de informação sobre } \\
\text { medicamentos } \\
\text { (5) Ausência de sistemas de identificação do paciente (pulseira } \\
\text { identificadora, etc) } \\
\text { (6) Sistemas de comunicação / informação deficientes } \\
\text { (7) Falta ou falha no processo de reconciliação } \\
\text { (8) Medicamento não disponível } \\
\text { (9) Condições de armazenamento inadequadas (falta de espaço, } \\
\text { etc) } \\
\text { (10) Sistemas deficientes de preparação / dispensação de } \\
\text { medicamentos } \\
\text { (11) Falta de informação, aos pacientes, sobre os medicamentos } \\
\text { (12) Falta de programas ou protocolos de acompanhamento } \\
\text { aos pacientes } \\
\text { (13) Falta de programas de assistência para pacientes } \\
\text { ambulatoriais (geriátricos, etc) } \\
\text { (14) Pessoal } \\
\text { (15) Fatores ambientais } \\
\text { (16) Situação de emergência } \\
\text { (17) Inércia do sistema } \\
\text { (18) Outros fatores }\end{array}$ \\
\hline
\end{tabular}

Fonte: adaptado de Otero López et al. (2008a)

Em síntese, o erro pode ser classificado dentre, pelo menos, 16 (dezesseis) diferentes tipos que não somente consideram a troca equivocada de medicamentos, mas também aspectos como a administração de dose ou via erradas e problemas relacionados ao armazenamento, à identificação do paciente e à monitorização do tratamento. Entre as causas do erro, as confusões na comunicação e na interpretação de informações são as mais recorrentes, assim como problemas na dispensação, no preparo e na administração de medicamentos. Também questões de ordem individual relativas, por exemplo, às condições de trabalho dos profissionais atuantes no sistema. 
O erro de medicação é um fato do qual nenhum hospital está imune (Werner, Nelson e Boehm-Davis, 2012). Neste sentido, a criação de uma cultura de segurança é um antecedente importante para reduzir a probabilidade da ocorrência do erro (Schneider, 2007). É preciso considerar que erros acontecem, em geral, em consequência de fatores decorridos em série, resultantes de ações partes de um sistema. Especialmente em ambiente hospitalar, onde múltiplas questões estão envolvidas para que ocorra o procedimento de administração do medicamento, uma ação equivocada pode vir de uma parte e afetar o todo, assim como a falta de estratégia no todo pode desencadear problemas aparentemente pontuais (Moreira Reis et al., 2010; Almeida Lopes et al., 2012).

Assim, o trabalho de prevenção do erro aplicado em pontos específicos do hospital tende a contribuir para a segurança do paciente e, portanto, deve ser uma constante. Para tanto, no Brasil, o Ministério da Saúde (Brasil, 2013) sugere que haja a "aplicação sistêmica e contínua de políticas, procedimentos, condutas e recursos" visando a "identificação, análise, avaliação, comunicação e controle de riscos e eventos adversos que afetam a segurança, a saúde humana, a integridade profissional", assim como "o meio ambiente e a imagem institucional". Tais focos voltam-se para a cultura da segurança, sendo esta o "conjunto de valores, atitudes, competências e comportamentos que determinam o comprometimento com a gestão da saúde e da segurança". O preceito é substituir "a culpa e a punição pela oportunidade de aprender com as falhas e melhorar a atenção à saúde".

\section{Identificação de setor para trabalho de prevenção}

A compreensão do sistema de medicação foi o primeiro passo para identificação de um setor do hospital onde, por meio da gestão de design, um projeto pudesse ser implantado, corroborando para o trabalho de prevenção. O sistema de medicação se refere a uma série de procedimentos que configuram o ciclo do medicamento no hospital (Monteschi Souta, 2015; Grou Volpe, 2014; Mcleod et al., 2014; Otero López et al., 2008a e 2008b; Perufo Opitz, 2006). A prescrição, a dispensação, o preparo e a administração de medicamentos são alguns dos principais processos constituintes desse sistema.

Em cada um dos processos do sistema de medicação, atuam profissionais - entre eles médicos, enfermeiros e farmacêuticos - que envolvem-se em atividades específicas para que a medicação chegue até o paciente. Neste contexto, "a escolha de um sistema de dispensação adequado contribuirá para a segurança e cumprimento da terapia medicamentosa prescrita ao paciente" (Ferreira Braga, 2014, p. 83). A dispensação é o processo de distribuição do medicamento, "a maneira pela qual a farmácia envia os medicamentos aos pacientes, mediante análise prévia das prescrições médicas" (Andrade dos Santos, 2006, p. 149), fazendo com que estes cheguem até a enfermaria para que seja administrado no paciente.

O fluxo de dispensação pode variar de hospital para hospital. No Brasil, destacam-se especialmente três: o sistema coletivo de dispensação, o individualizado e o de dispensação em dose unitária (Ferreira Braga, 2014; Maiques Juliani, 2014; Andrade dos Santos, 2006). O que varia entre eles é o curso de trabalho entre os setores, ou seja, as atividades desempenhadas pelos profissionais envolvidos - médicos, farmacêuticos, enfermeiros, entre outros - para que a medicação chegue até o paciente (Figura 2). 
Farmácia hospitalar como oportunidade para a gestão de design no trabalho de prevenção do erro de medicação: estudio em uma realidade brasileira.

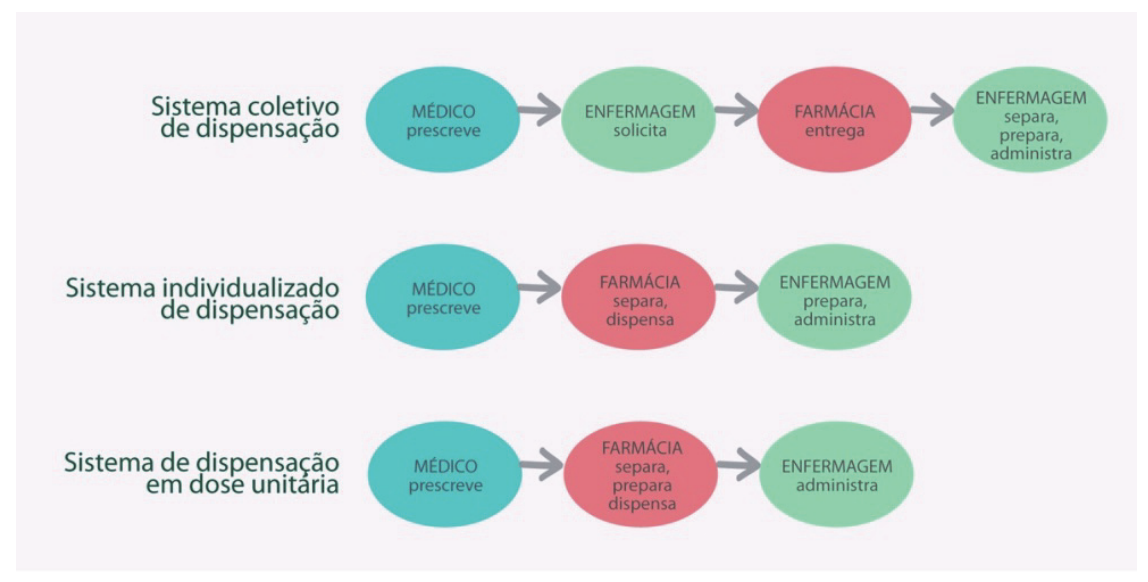

Fig. 2 - Fluxos do sistema de medicação hospital. Fonte: os autores

A prescrição é a indicação do medicamento que configurará o tratamento do paciente e é realizada pela equipe médica. Por meio de receituários, os medicamentos são requeridos à farmácia diretamente pelo médico ou com solicitação realizada via enfermagem. A farmácia entrega os medicamentos passando pelo processo de separação, que engloba a leitura da prescrição e a dispensação de medicamentos fracionados - a "subdivisão de um medicamento em frações individualizadas" (Brasil, 2006, p. 4). No sistema coletivo de dispensação, a enfermagem fica responsável, também, pela separação do medicamento que irá administrar. O processo de preparação do medicamento é, em geral, uma tarefa da enfermagem - exceto no sistema de dispensação em dose unitária, quando a própria farmácia realiza o preparo - sendo, os enfermeiros, os responsáveis pela administração do medicamento no paciente.

Analisados os fluxos que configuram os sistemas de medicação na realidade brasileira, levantou-se, ainda, os pontos do processo a serem considerados para a prevenção do erro de medicação. Nesse sentido, identificou-se que a Anvisa (2010) ressalta ações que perpassam pelos processos de prescrição, de dispensação, de administração, de monitorização e de sistemas e gerenciamento do controle (Quadro 2). 
Quadro 2. Prevenção nos processos intrínsecos ao sistema de medicação

\begin{tabular}{|c|c|}
\hline \multicolumn{2}{|r|}{ Pontos dos processos para prevenção do erro } \\
\hline Prescrição & $\begin{array}{l}\text { Avaliação da necessidade e seleçăo do medicamento correto; } \\
\text { Individualizaçăo do regime terapêutico; } \\
\text { Estabelecimento da resposta terapêutica desejada. }\end{array}$ \\
\hline Dispensação & $\begin{array}{l}\text { Revisăo da prescriçăo; } \\
\text { Processamento da prescriçáo; } \\
\text { Mistura e preparo dos medicamentos; } \\
\text { Dispensaçáo dos medicamentos de maneira adequada e oportuna. }\end{array}$ \\
\hline Administração & $\begin{array}{l}\text { Administraçăo do medicamento correto para o paciente correto; } \\
\text { Administraçăo do medicamento quando indicado; } \\
\text { Informaçăo ao paciente sobre a medicaçáo; } \\
\text { Inclusăo do paciente no processo de administraçáo. }\end{array}$ \\
\hline Monitorização & $\begin{array}{l}\text { Monitorizaçáo e documentaçăo da resposta do paciente; } \\
\text { Identificaçăo e notificaçáo de eventos adversos aos medicamentos; } \\
\text { Reavaliação da seleçáo do medicamento, regime, frequência e } \\
\text { duraçáo do tratamento. }\end{array}$ \\
\hline $\begin{array}{l}\text { Sistemas e } \\
\text { gerenciamento do } \\
\text { controle }\end{array}$ & $\begin{array}{l}\text { Colaboraçăo e comunicaçăo entre os responsáveis pelos cuidados } \\
\text { de saúde; } \\
\text { Revisão e gerenciamento do regime farmacoterapêutico do } \\
\text { paciente. }\end{array}$ \\
\hline
\end{tabular}

Fonte: adaptado de Anvisa (2010)

Com levantamento dos pontos em que a prevenção do erro de medicação pode ser aplicada no sistema e com o cruzamento dessas informações junto às características de trabalho dos setores do hospital, a farmácia ficou evidenciada como mote estratégico. Dentre os processos de prescrição, dispensação, administração, monitorização e gerenciamento do controle, a farmácia revelou-se como setor-chave para início de um trabalho de prevenção via gestão de design, especialmente porque o profissional que atua na farmácia tem posição estratégica para supervisionar a qualidade do processo de medicação, desde a prescrição até a distribuição (Guchelaar et al., 2005).

Tamuz, Thomas e Franchois (2004) esclarecem que o fluxo de dados que levam ao erro de medicação pode ser reduzido por meio de rotinas de aprendizagem organizacional implantadas na farmácia hospitalar. A farmácia é um ponto importante na prevenção do erro no hospital, visto que o potencial de erro de medicação existe em diferentes setores, mas problemas nos procedimentos da farmácia podem estender-se para as demais fases do sistema de medicação (Almeida Lope et al., 2012).

Pela amplitude dos serviços que são de responsabilidade da farmácia hospitalar, é entendido que ela se caracteriza como uma unidade técnico-administrativa dentro do hospital, sendo que suas funções a relacionam com outros setores (Figura 3) do hospital (Andrade dos Santos, 2006). Dentre as funções da farmácia hospitalar, estão os processos de gerenciamento, de seleção de medicamentos, de programação, de aquisição, armazenamento, distribuição e de informação e a responsabilidade sobre a farmacotécnica no qual se inserem os procedimentos de fracionamento - o ensino e a pesquisa (Cavalcante Dantas, 2011). 
Farmácia hospitalar como oportunidade para a gestão de design no trabalho de prevenção do erro de medicação: estudio em uma realidade brasileira.

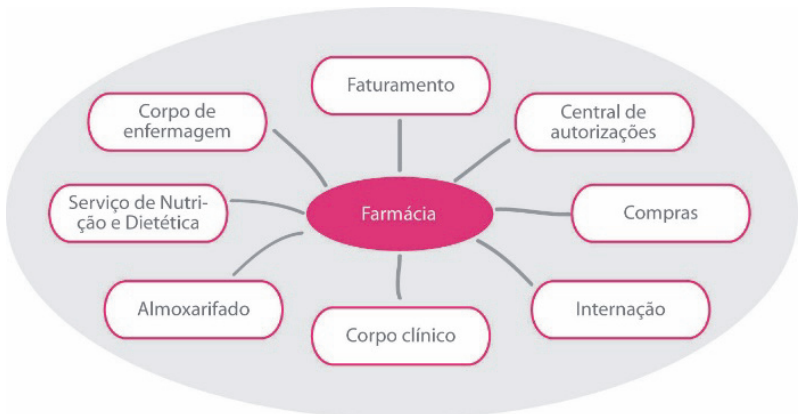

Fig. 3 - Relações da farmácia no context hospitalar. Fonte: adaptado de Andrade dos Santos (2006, p. 53)

Dentre os citados, o armazenamento, o fracionamento e a distribuição ou dispensação são procedimentos rotineiros de uma farmácia hospitalar. E, conforme destaca Almeida Lopes et al. (2012), embora exista risco de erro nos demais setores do hospital, a farmácia é o setor de dispensação responsável pelo quantitativo de medicamentos. Portanto, erros ocorridos na farmácia fazem com que o risco se estenda para as demais fases do sistema de medicação, o que pode ser agravado mediante o quantitativo de pacientes sob os cuidados da equipe multidisciplinar.

Azevedo Anacleto et al. (2010, p. 9) enfatiza que, como a principal função da farmácia é a dispensação nas quantidades e especificações solicitadas e nos prazos requeridos, ela promove o uso seguro e correto de medicamentos. Destaca que "a organização e sua prática devem prevenir que erros de dispensação aconteçam e por criarem oportunidades de erros de administração, possam atingir os pacientes". Neste sentido, a farmácia é, estrategicamente, um setor do hospital cuja prevenção do erro impacta em todo o sistema de medicação.

Visando a prevenção do erro, a aplicação da gestão de design na farmácia hospitalar pode, contudo, se dar a partir de ações diversas. A pesquisa demonstrou que, dentre os principais enfoques do erro na farmácia dos hospitais, estão o erro de conteúdo, o erro de rotulagem e o erro de documentação. Observando as características de cada um e os exemplos e, ainda, visitando a realidade de um hospital brasileiro, foi também possível apontar algumas ações de design para corroboração na prevenção (Quadro 3). 
Quadro 3. Características, exemplos e apontamentos de possíveis ações de design

\begin{tabular}{|c|c|c|}
\hline $\begin{array}{l}\text { Tipos de erro de erro } \\
\text { dispensação }\end{array}$ & $\begin{array}{l}\text { Exemplos a partir da } \\
\text { farmácia hospitalar }\end{array}$ & $\begin{array}{c}\text { Possíveis ações de design } \\
\text { para prevenção }\end{array}$ \\
\hline $\begin{array}{l}\text { Erro de conteúdo } \\
\text { Erro relativo aos } \\
\text { medicamentos prescritos que } \\
\text { foram erroneamente } \\
\text { dispensados. }\end{array}$ & $\begin{array}{l}\text { Exemplos de erro de conteúdo } \\
\text { Dispensaçăo de medicamento } \\
\text { diferente do prescrito; } \\
\text { Dispensação de medicamento não } \\
\text { prescrito; } \\
\text { Dispensação de medicamento com } \\
\text { outra forma farmacêutica ou outra } \\
\text { concentração; Excesso ou omissão } \\
\text { de dose ao dispensar; } \\
\text { Medicamentos dispensados } \\
\text { mesmo que visivelmente com } \\
\text { desvio de qualidade; } \\
\text { Medicamentos dispensados sem } \\
\text { que constem todos os dados } \\
\text { obrigatórios na prescrição; }\end{array}$ & $\begin{array}{l}\text { Prevenção do erro de conteúdo } \\
\text { Layout das tabelas e fichas de requisição e dispensação; } \\
\text { Sinalização dos diferentes produtos em prateleira; } \\
\text { Layout de etiqueta para identificação e diferenciação de produtos; } \\
\text { Identificação de pacientes e/ou enfermarias; } \\
\text { Diferenciação de indicativos de dose; } \\
\text { Estocagem com controle otimizado de validade; } \\
\text { Sistemas integrados entre os setores desde a prescrição. }\end{array}$ \\
\hline $\begin{array}{l}\text { Erro de rotulagem } \\
\text { Erro provindo de dados } \\
\text { informacionais no rótulo do } \\
\text { medicamento, seja pela } \\
\text { incorreta identificação, seja } \\
\text { por erros de grafia ou por } \\
\text { ilegibilidade no próprio } \\
\text { produto ou nas etiquetas } \\
\text { utilizadas pela farmácia. }\end{array}$ & $\begin{array}{l}\text { Exemplos de erro de rotulagem } \\
\text { Nome errado do medicamento; } \\
\text { Concentraçäo ou forma } \\
\text { farmacêutica apresentada } \\
\text { erroneamente; } \\
\text { Data incorreta; } \\
\text { Orientaçōes erradas quanto ao uso } \\
\text { ou armazenamento; }\end{array}$ & $\begin{array}{l}\text { Prevenção do erro de rotulagem } \\
\text { Cor das etiquetas e rótulos; } \\
\text { Tamanho das etiquetas e rótulos; } \\
\text { Modos de escrita voltados para legibilidade e leiturabilidade; } \\
\text { Sistemas que integrem todo o processo da farmácia; } \\
\text { Processos que facilitem a identificação e checagem; } \\
\text { Distinção de produtos fracionados enão fracionados; } \\
\text { Sinalizaçōes orientadas à validade do produto estocado; } \\
\text { Indicativos claros sobre a forma de armazenamento do produto; } \\
\text { Layout das orientaçōes de uso. }\end{array}$ \\
\hline $\begin{array}{l}\text { Erro de documentação } \\
\text { Relacionados com o registro } \\
\text { de dados incorretos na } \\
\text { documentaçāo de } \\
\text { dispensação. }\end{array}$ & $\begin{array}{l}\text { Exemplos de erro de } \\
\text { documentação } \\
\text { Ausência ou erro no registro de } \\
\text { medicamentos controlados; } \\
\text { Ausência de data ou de assinatura } \\
\text { do prescritor ou do dispensador. }\end{array}$ & $\begin{array}{l}\text { Prevenção do erro de documentação } \\
\text { Identificação de áreas exclusivas para medicamentos controlados; } \\
\text { Sinalizaçōes especificas nas fichas de requisiçăo; } \\
\text { Sistema de comunicação entre os funcionários; } \\
\text { Layout que facilite a identificação, o controle, a checagem e o } \\
\text { arquivamento de documentos. }\end{array}$ \\
\hline
\end{tabular}

Fonte: adaptado de Azevedo Anacleto et al. (2010) - erros e exemplos. Os autores - apontamentos de ações.

As ações apontadas são práticas de design que se encontram associadas aos níveis da gestão de design. Esses níveis são o operacional, o tático e o estratégico, nos quais o design influencia, respectivamente, na oferta, nas pessoas e na organização (MOZOTA; KLÖPSCH; COSTA, 2011). Na gestão de design operacional, o grau de aplicação se dá nas atividades primárias, como ações de melhoria no layout das etiquetas aplicadas nos medicamentos fracionados. Na gestão de design tático, como competência administrativa, o design muda atividades de apoio, tais como integrações dos sistemas que venham a permitir a comunicação plena entre os setores envolvidos no sistema de medicação. Na gestão de design estratégico, o design pode transformar a cadeia de valor do setor. Neste último, as ações de design aplicadas à farmácia podem ser, futuramente, os meios que venham a operar para novos modelos de gestão na área e, inclusive, para a revisão e melhoria de protocolos e outros aspectos da legislação que regula o sistema de medicação brasileiro.

\section{Conclusão}

Considerando que o erro de medicação é um fator que nenhum hospital está imune e, portanto, as ações de prevenção visando a segurança são requeridas entre todas as atividades hospitalares, este estudo 
Farmácia hospitalar como oportunidade para a gestão de design no trabalho de prevenção do erro de medicação: estudio em uma realidade brasileira.

evidenciou que a gestão de design tem atributos que permitem subsidiar este meio. $O$ contexto investigado revela que a taxa de erros de medicação na realidade brasileira requer atenção. Juntamente, os registros da literatura e dos órgãos competentes no país mencionam ações para a segurança do sistema de medicação. A pesquisa mostrou que o Design pode contribuir para tanto, especialmente porque as algumas das ações de prevenção estão adequadas ao escopo da gestão de design.

Respondendo ao questionamento sobre qual setor do hospital iniciar um trabalho de gestão de design que venha a contribuir para a prevenção do erro de medicação, a pesquisa apontou para a farmácia hospitalar. Por meio do levantamento sistemático, ficou evidenciado que este setor é estratégico no contexto do sistema de medicação. Dentre as principais formas de dispensação de medicamentos na realidade dos hospitais brasileiros, a farmácia hospitalar tem função central no sistema, pois é ela que controla o estoque e o armazenamento, o registro, a separação e a distribuição dos medicamentos.

É na farmácia hospitalar que o sistema de medicação é gerenciado nos seus aspectos técnicos e administrativos. Torna-se fundamental que ações para prevenção do erro de medicação sejam aplicadas diretamente no contexto da farmácia e em todos os níveis de suas atividades. Erros de conteúdo, de rotulagem e de documentação são alguns dos tipos que podem partir da farmácia hospitalar e atingir o sistema como um todo, chegando a equívocos que impliquem no paciente.

Por meio de um trabalho que englobe a gestão de design na farmácia hospitalar, ações que apoiem o trabalho de prevenção podem ser implantadas a curto, médio e longo prazo. Atividades que, hoje, são em geral desenvolvidas por farmacêuticos - tais como layout para identificação de medicamentos fracionados e layout de controle de estoque e checagem - e que fazem parte das habilidades de Design, podem ser melhoradas, otimizadas e, com isso, mais adequadas à prevenção do erro.

Dentre os apontamentos de ações a serem implantadas pela gestão de design visando a prevenção do erro de medicação, estão possibilidades nos níveis operacional, tático e estratégico. A identificação dos produtos pela melhoria da legibilidade e leiturabilidade, a indicação de aspectos que promovam a adequada comunicação entre os profissionais envolvidos no sistema de medicação e, por consequência, a manifestação de modelos de gestão voltados especificamente à farmácia hospitalar são, respectivamente, exemplos da aplicação da gestão de design neste meio.

Esta pesquisa é parte de trabalhos ligados ao projeto "Design e Saúde: da saúde do paciente às questões de saúde do trabalhador", em prática no Núcleo de Gestão de Design e Laboratório de Design e Usabilidade da Universidade Federal de Santa Catarina. Trata-se da primeira parte de um estudo sobre a medicação em hospitais, tendo se limitado especialmente ao contexto revelado pelo levantamento sistemático focado em literatura. No entanto, a partir desta pesquisa, projetos aplicados já estão em prática em uma farmácia hospitalar, contando com o apoio de uma equipe multidisciplinar, que conta com pesquisadores designers, em parceria com profissionais da área da saúde atuantes em um hospital público psiquiátrico. Futuros estudos focam em demonstrar os resultados dos projetos aplicados e os ganhos da prevenção do erro de medicação por meio da gestão de design. 


\section{Referências}

ALMEIDA, D. et al. (2012). "Análise da rotulagem de medicamentos semelhantes: potenciais erros de medicação" en Revista da Associação Médica Brasileira, São Paulo, vol. 58, issue 1, p.95-103.

ANDRADE, G. A. (2006). Gestão de farmácia hospitalar. São Paulo: Senac São Paulo.

ANVISA (2010). Agência Nacional de Vigilância Sanitária. Erro de medicação: Informe SNVS/Anvisa/Nuvig/Gfarm $n^{o} 04$ de 7 de dezembro de 2010. Brasília: Ministério da Saúde do Brasil <http://portal.anvisa.gov.br>. [Consulta: 29 de setiembre de 2015].

AZEVEDO ANACLETO, T. et al. (2010). "Erros de medicação" en Farmácia Hospitalar: Encarte, São Paulo, jan/fev. 2010, p. 1-24.

BEST, K. (2012). Fundamentos de gestão do design. Porto Alegre: Bookman.

BLUM, A., MERINO - DÍAZ, E. y MERINO - SCHMIDT, G. (2015). "Método visual para revisão sistemática em design com base em conceitos da mineração de dados" en IV International Conference On Integration Of Design, Engineering And Manegement For Inovation. Florianópolis: Udesc. 278-289.

BORGES ROSA, M. y PERINI, E. (2003). "Erros de medicação: quem foi?" en Revista da Associação Médica Brasileira, vol. 49, issue 3, p. 335-341.

BORJA DE MOZOTA, B., KLÖPSCH, C. y XAVIER DA COSTA, F. C. (2011). Gestão do design: usando o design para construir valor de marca e inovação corporativa. Porto Alegre: Bookman.

BRASIL. Ministério da Saúde. Agência Nacional de Vigilância Sanitária. Resolução da Diretoria Colegiada. $R D C n^{o}$ 36, institui ações para a segurança do paciente em serviços, 25 de julho de 2013.

BRASIL. Ministério da Saúde. Agência Nacional de Vigilância Sanitária. Resolução da Diretoria Colegiada. $R D C n^{o}$ 214, dispõe sobre boas práticas de manipulação de medicamentos para uso humano em farmácias, de 18 de dezembro de 2006 .

CAVAlCANTE, S. (2011). "Farmácia e Controle das Infecções Hospitalares" em Farmácia Hospitalar. fev. 2011, Brasília, p. 1-20.

EBSCO-PUBLISHING. (2015) Ebsco Host: Publicações cientificas. <https://search.ebscohost.com/>. [Consulta: 24 setiembre 2015].

ELSEVIER. (2015a). Science Direct. <www.sciencedirect.com>. [Consulta: 24 setiembre2015].

ELSEVIER. (2015b). Scopus. <http://www.scopus.com/>. [Consulta: 24 setiembre2015].

FAPESP et al. (2015). Scielo: Scientific Eletronic Library Online. $<$ http://www.scielo.org/>. [Consulta: 24 setiembre 2015].

FERREIRA BRAGA, R. (2014). "Dispensação de medicamentos” en Ferreira Braga, R. (org.). ABC da farmácia hospitalar. São Paulo: Atheneu.

GROU VOLPE, C. R. (2014). Eventos adversos no sistema de medicação: a magnitude do problema. Tese de Doutorado. Brasília: Universidade de Brasília.

GUCHELAAR, H. et al. (2005). "Medication errors: hospital pharmacist perspective" en Drugs, vol. 65, issue 13, p.1735-1746.

IBICT. (2015). BDTD: Biblioteca Digital de Teses e Dissertações. <http://bdtd.ibict.br/>. [Consulta: 25 setiembre 2015].

MAIQUES, R. (2014). Organização e funcionamento de farmácia hospitalar. São Paulo: Érica.

MARTIN, C. y GADBOIS, C. (2007). “A ergonomia no hospital” en Falzon, P. (Ed.). Ergonomia. São Paulo: Blucher.

MCLEOD, M. et al. (2014). "A national survey of inpatient medication systems in English NHS hospitals" en Bmc Health Services Research, vol. 14, issue 1, p. 93-104.

MENDES, A. E. M. et al. (2014). "Erros de medicação: uma abordagem para clínicos” en Revista Médica da UFPR, vol. 1, issue 4, p. 169-172.

MONTESCHI, M. (2015). Sistemas de medicação e erros em unidades de psiquiatria de um município paulista. Tese de Doutorado. Ribeirão Preto: Universidade de São Paulo. 
Farmácia hospitalar como oportunidade para a gestão de design no trabalho de prevenção do erro de medicação: estudio em uma realidade brasileira.

MOREIRA, A. M. (2010). "Errors in medicine administration - profile of medicines: knowing and preventing" en Acta Paulista de Enfermagem, vol. 23, issue 2, p.181-186.

NCC MERP (2010). National Coordinating Council for Medication Error Reporting and Prevention. About Medication Errors: What is a Medication Error? <http://www.nccmerp.org/about-medication-errors>. [Consulta: 29 setiembre 2015].

NCC MERP (1998). National Coordinating Council for Medication Error Reporting and Prevention. Taxonomy of medication errors. 1998. <http://www.nccmerp.org/sites/default/files/taxonomy2001-07-31.pdf>. [Consulta: 29 setiembre 2015].

OLIVEIRA, S. et al. (2005). "Mineração de dados" en Oliveira Rezende, S., Sistemas inteligentes: fundamentos e aplicações. Barueri: Manole.

OTERO, M. et al. (2008a). "Actualización de la clasificación de errores de medicación del grupo Ruiz-Jarabo 2000" en Farmacia Hospitalaria, vol. 32, issue 1, p.38-52.

OTERO, M. et al (2008b). "Evaluación de las prácticas de seguridad de los sistemas de utilización de medicamentos en los hospitales españoles (2007)" en Medicina Clinica, vol. 10, issue 5, p. 13-15.

PERUFO, S. (2006). Sistema de medicação: análise dos erros nos processos de preparo e administração de medicamentos em um hospital de ensino. Tese de Doutorado. Ribeirão Preto: Universidade de São Paulo.

REASON, J. (1990) Human error. Cambridge: Cambridge University Press.

SCHNEIDER, P. J. (2007). “Opportunities for pharmacy” en American Journal Of Health-system Pharmacy, vol. 69, issue 9, p. 10-16.

TAMUZ, M; THOMAS, \& J; FRANCHOIS, K e. "Defining and classifying medical error: lessons for patient safety reporting systems" en Quality And Safety In Health Care, vol. 13, issue 1, p.13-20.

THOMSON REUTERS. Web of Science. (2015). < http://www.webofscience.com>. [Consulta: 25 setiembre 2015].

WERNER, N. E., NELSON, E. T. y BOEHM-DAVIS, D. A. (2012). "Human factors methods to reduce medication error: using task analysis in a pediatric and adult pharmacy" en Work, vol. 41, p. 5665-5667. 